\title{
Very low flux adaptive optics using spatial and temporal priors
}

\author{
Clémentine Béchet ${ }^{1, a}$, Michel Tallon ${ }^{2}$, and Miska Le Louarn ${ }^{1}$ \\ 1 European Southern Observatory (ESO), Karl-Schwarzschild-Strasse 2, 85748 Garching, GERMANY \\ 2 Centre de Recherche Astrophysique de Lyon - Observatoire de Lyon, 9 Avenue Charles André 69561 \\ Saint Genis Laval cedex, FRANCE
}

\begin{abstract}
Although laser guide stars are great candidates to increase the sky coverage of adaptive optics (AO) systems, their use on an Extremely Large Telescope leads to difficulties that can be avoided by using natural guide stars (NGS). Therefore it is interesting to optimize the behavior of an AO system running on very faint NGS. Very low flux conditions with a Shack-Hartmann wavefront sensor (WFS) leads, according to Poisson statistics, to a certain amount of sub-apertures receiving no photons during each WFS exposure. Such extinctions are considered in current $\mathrm{AO}$ systems as measurements of null slopes. Even if the AO loop can still be closed with extinctions in a third of the sub-apertures or more, we show that spatial priors in the reconstruction are not sufficient to guarantee a good AO correction. Temporal priors become essential in this regime. New control approaches are investigated. It relies on a fast maximum a posteriori reconstruction which takes into account the extinctions occurred in every loop step. Temporal priors help to optimize the closed-loop correction. The resulting performance is compared to the classical matrix-vector multiplication with an integrator, thanks to end-to-end simulations.
\end{abstract}

\section{Introduction}

Although laser guide stars (LGS) are great candidates to increase the sky coverage of the adaptive optics (AO) systems, their use on an Extremely Large Telescope (ELT) leads to difficulties that can be avoided by using natural guide stars (NGS). On an ELT, the tremendous cone effect [1], the elongated spot on the Shack-Hartmann wavefront sensor, the tip-tilt undetermination [2] are the main issues one has to overcome in order to use LGSs. It is thus interesting to optimize the behavior of an AO system running on faint NGS.

In the framework of the European ELT Design Study, fast algorithms for reconstruction and control have been studied, in order to derive solutions to the severe computational requirements for large AO systems such as the one expected for the European ELT. Thanks to end-to-end simulations, Béchet et al. have shown that the Fractal Iterative Method with an internal model control (FrIM-IMC in [3]) performs as well as a classical matrix-vector multiply reconstructor with an integrator in a large range of conditions, except in very low flux situations. This observation has led to the study presented here.

Since the Fractal Iterative Method (FrIM) [4] provides a maximum a posteriori (MAP) reconstruction thanks to the use of prior knowledge of the turbulence statistics, it is expected to outperform any other reconstructors. Particularly, this advantage increases when the measurement noise becomes larger [5], and so when the guide star gets fainter. However, in a closed-loop AO system running around its limiting magnitude regime, the spatial priors included in the MAP reconstruction are not enough to guarantee a good correction. In the low flux regime, the control becomes a key point, because it includes the temporal modeling or priors assumed on the turbulence. The low flux regime thus provides interesting conditions to assess the quality of the control.

We present here the first results of a study for AO with such very low flux, using the Fractal Iterative Method (FrIM) for the reconstruction step. Several reconstruction and control algorithms are compared and end-to-end simulations enlighten the reachable performance. First, in Sect. 2, the simulated conditions are presented and the performance of the classical integrator is compared to the FrIM-IMC strategy used in Béchet et al. [3]. In Sect. 3, alternative ways to use the spatial priors in the reconstructor are investigated. Several noise models based on the flux variations are inserted in

\footnotetext{
a e-mail: cbechet@eso.org
}

This is an Open Access article distributed under the terms of the Creative Commons Attribution-Noncommercial License, which permits unrestricted use, distribution, and reproduction in any noncommercial medium, provided the original work is properly cited. 
Fig. 1.

Telescope and AO parameters for the simulations.

\begin{tabular}{ll}
\hline Diameter & $D=42 \mathrm{~m}$ \\
\hline Central obstr. & $0.28 D$ \\
\hline SH sub-apertures & $84 \times 84$ \\
\hline \# slopes & 10080 \\
\hline \# commands & 5402 \\
\hline AO loop delay (2 frames) & $\tau=4 \mathrm{~ms}$ \\
\hline AO wavelength & $\lambda_{\mathrm{AO}}=700 \mathrm{~nm}$ \\
\hline imaging wavelength & $\lambda_{I}=2.2 \mu \mathrm{m}$ \\
\hline sub-apertures FOV & $2.4 \mathrm{arcseconds}$ \\
\hline CCD pixels / sub-ap. & $6 \times 6$ \\
\hline read-out noise & $0 \mathrm{e}-$ \\
\hline \# photons / frame / sub-ap. & 1 \\
\hline Atm. coherence time & $\tau_{0}=3 \mathrm{~ms}$ \\
\hline Atm. outer scale & $L_{0}=25 \mathrm{~m}$ \\
\hline Atm. Fried parameter & $r_{0}=0.13 \mathrm{~m}$ \\
\hline
\end{tabular}

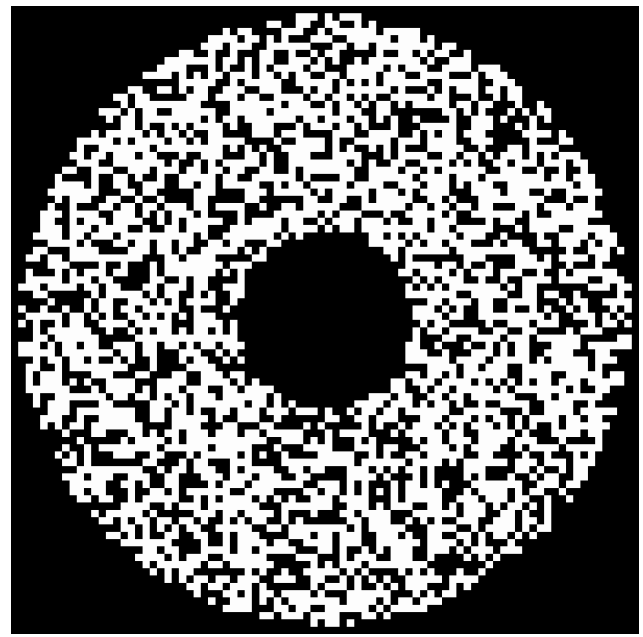

Fig. 2. Example of distribution of the $63 \%$ illuminated sub-apertures (white) over the pupil for a guide star flux corresponding to an average of 1 photon per frame per sub-aperture.

FrIM-IMC algorithm and compared, as an attempt to improve the performance. Finally, in Sect. 4, a pseudo open-loop control [6] is studied to efficiently combine the use of spatial and temporal priors.

\section{Very low flux performance : the classical method as a reference}

End-to-end simulations of a typical single-conjugate AO for the European ELT are considered in this paper. The parameters of the system are summarized in the table of Fig. 1. Except for the AO loop frequency which is here $500 \mathrm{~Hz}$, all the remaining parameters are identical to the ones used by Béchet et al.[3]. In the simulations of this study, an average of 1 photon per sub-aperture per frame is received from the guide star. According to Poisson statistics, this means that, at every new frame, $37 \%$ of the sub-apertures will not receive any photon. Figure 2 shows a realization of a Shack-Hartmann wavefront sensor (WFS) exposure, where sub-apertures that have received at least one photon are enlighten in white, the others are blacked out. Such extinctions inside the annular pupil are considered in current AO systems as measurements of null slopes, to which we refer hereafter as artificial measurements.

Figure 3 shows the long-exposure Strehl convergence (integrated from the 30-th loop on) of three algorithms. The dotted line refers to a regularized reconstructor applied as a matrix-vector multiply (MVM) and combined with an integrator. We refer hereafter to this algorithm as MVM-I. The reconstructor is deduced from a calibrated interaction matrix. This algorithm is representative of what is currently implemented on existing AO systems, and it is thus considered as a reference here. However, it is not a fast algorithm adapted to a real-time $\mathrm{AO}$ of this size. The dashed line is also obtained with an integrator but the reconstructor has been exchanged for the Fractal Iterative Method (FrIM) [4], which is a fast maximum a posteriori reconstruction algorithm. In this case, the reconstructor is directly applied on the measurements corresponding to the residual wavefront, although the priors correspond to the incident one. The convergence behavior of this algorithm, referred hereafter as FrIM-I, is different at the start but the final performance is similar to MVM-I, close to a Strehl of $30 \%$. Last, the solid line stands for a FrIM reconstruction associated to a particular internal model control (FrIM-IMC) [3] 


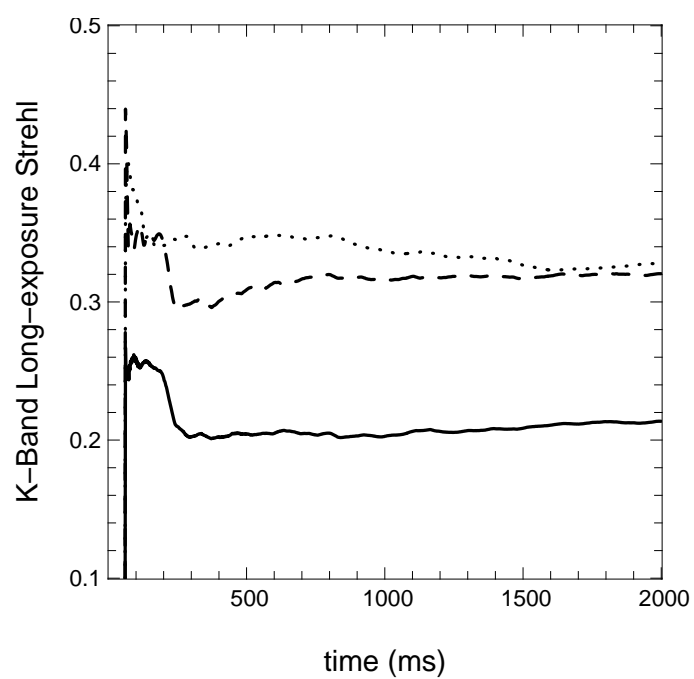

Fig. 3. Long-exposure Strehl at $2.2 \mu \mathrm{m} v s$ simulation time. The value at time $t$ results from an integration from $60 \mathrm{~ms}$ up to time $t .3$ algorithms are compared. Dotted : MVM-I. Dashed : FrIM-I. Solid : FrIM-IMC.

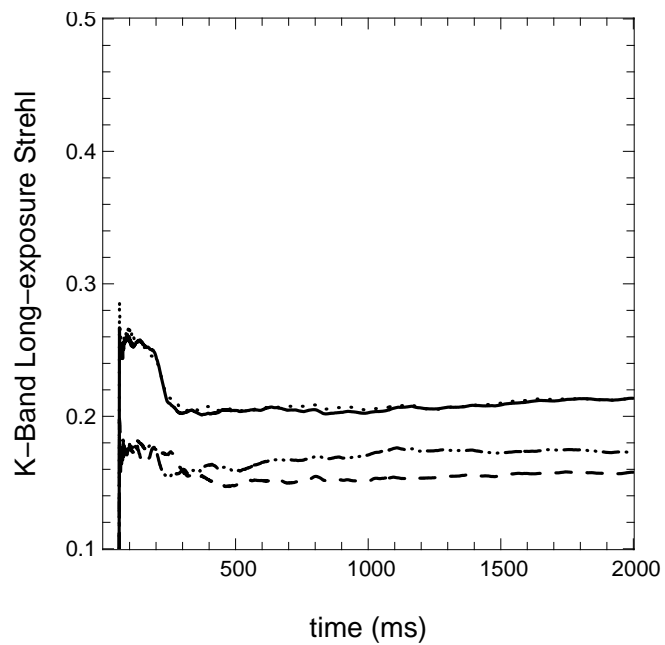

Fig. 4. Same axes as Fig. 3. Control algorithm is FrIM-IMC. Four different noise models are used in FrIM. Solid: uniform noise (as in Fig. 3). Dotteddashed : $\gamma=0.1$. Dashed $: \gamma=0$. Dotted $: \gamma=1$.

[7], instead of the integrator. While this recent algorithm demonstrates similar performance as MVMI does in main simulated conditions reported by Béchet et al.[3], the reached Strehl appears really poor in low flux conditions. Two aspects of the algorithm are modified in the next sections in order to improve this: the use of the spatial priors and the control scheme for the temporal filtering.

\section{On the use of spatial priors with FrIM-IMC at low flux}

The algorithm FrIM-IMC is based upon a MAP wavefront reconstructor, which can be written

$$
\mathbf{R}_{\mathrm{MAP}}=\left(\mathbf{S}^{\mathrm{T}} \cdot \mathbf{C}_{\boldsymbol{e}}^{-1} \cdot \mathbf{S}+\mathbf{C}_{\boldsymbol{w}}^{-1}\right)^{-1} \cdot \mathbf{S}^{\mathrm{T}} \cdot \mathbf{C}_{\boldsymbol{e}}^{-1}
$$

where $\mathbf{S}$ and $\mathbf{S}^{\mathrm{T}}$ are the matrix of the linear model for the wavefront sensing process and its transposed form and $\mathbf{C}_{e}$ is the covariance matrix of the measurement noise. The covariance matrix of the turbulent wavefront $\mathbf{C}_{w}$ is the a priori information inserted in the reconstructor, based on turbulence statistics. For a NGS, $\mathbf{C}_{\boldsymbol{e}}^{-1}$ is a diagonal matrix

$$
\mathbf{C}_{\boldsymbol{e}}^{-1}=\operatorname{diag}\left(1 / \sigma_{i}^{2}\right)=\operatorname{diag}\left(\omega_{i}\right)
$$

where $\sigma_{i}^{2}$ is the noise variance on the $i$-th measurement and $\omega_{i}$ is the weight applied on this data in the reconstructor. The noise is considered to be the difference between the data coming from the simulator and noiseless data computed from the WFS model $\mathbf{S}$. A known distorted wavefront is used to estimate, somehow calibrate, the variance of the noise or the weight. We investigated 4 different strategies to model the data weighting in the FrIM reconstructor. The first one consists in writing

$$
\omega_{i}=\omega_{\left\langle n_{\mathrm{ph}}\right\rangle}(1) \quad \forall i
$$

where $\omega_{\left\langle n_{\mathrm{ph}\rangle}\right\rangle}$ represents the weight as a discrete function of the average number of photons received per frame and per sub-aperture $\left\langle n_{\mathrm{ph}}\right\rangle$. This leads to a uniform noise in $\mathbf{C}_{\boldsymbol{e}}$ and the artificial measurements are considered by the reconstructor to be as reliable as the data coming from the analysis of one or several detected photons. This is what the reconstructors presented in Fig. 3 implicitly do. 
It is also possible to express the weight as a function $\omega_{n_{\mathrm{ph}}}$ of the number of photons $n_{\mathrm{ph}}$ effectively received in each frame. Then, for each measurement, the exact weigth corresponding to the actual number of photons received in each sub-aperture in each frame can be used. This is made possible using FrIM reconstructor because $\mathbf{C}_{\boldsymbol{e}}^{-1}$ can be updated on the fly, according to the instantaneous flux in each sub-aperture. The simplest way for this consists in updating at every loop $\mathbf{C}_{\boldsymbol{e}}^{-1}$ with

$$
\omega_{i}=\omega_{n_{\mathrm{ph}}}\left(p_{i}\right)
$$

where $p_{i}$ is the number of photons received by the sub-aperture producing the $i$-th measurement. An extinction means a lack of information for the reconstruction, that is to say $\omega_{n_{\mathrm{ph}}}(0)=0$. In such case, the wavefront reconstruction is thus applied with 1/3 less data (distributed as in Fig. 2 over the pupil for instance), but the statistical description of the noise is more accurate. Two other noise descriptions to build $\mathbf{C}_{\boldsymbol{e}}^{-1}$ from Eq. (2) have also been tested, which are based on

$$
\omega_{i}=\left\{\begin{array}{ll}
\omega_{n_{\mathrm{ph}}}\left(p_{i}\right) & \text { if } \mathrm{p}_{\mathrm{i}} \neq 0 \\
0.1 \omega_{n_{\mathrm{ph}}}(1) \text { if } \mathrm{p}_{\mathrm{i}}=0
\end{array} \quad \text { and } \quad \omega_{i}= \begin{cases}\omega_{n_{\mathrm{ph}}}\left(p_{i}\right) & \text { if } \mathrm{p}_{\mathrm{i}} \neq 0 \\
\omega_{n_{\mathrm{ph}}}(1) & \text { if } \mathrm{p}_{\mathrm{i}}=0\end{cases}\right.
$$

where $p_{i}$ still is the instantaneous number of photons received for the $i$-th measurement. The four models described above are referred in this paper as uniform noise model, $\gamma=0, \gamma=0.1$ and $\gamma=1$ respectively, since $\gamma$ is the factor applied on the maximum weight when extinctions occur $\left(p_{i}=0\right)$. It can be interpreted as giving more or less importance to the artificial measurements in the reconstruction. From another point of view, this means also giving more or less importance to the spatial priors in the reconstructor to deduce the wavefront from interpolation.

All these noise models have been tested for FrIM-IMC algorithm. The comparison is plotted in Fig. 4. The solid line stands for the uniform noise case, as in Fig. 3. The dashed line shows the reached Strehl ratio when null weight, $\gamma=0$ (infinite noise level), is assumed on extinctions. This second model does not improve the method, it even provides worse result. Using $\gamma=0.1$ leads to a slightly better Strehl (dotted-dashed), than $\gamma=0$. Finally, with $\gamma=1$, the behavior is very similar to the uniform noise case. This can be explained because $74 \%$ of the sub-apertures, according to Poisson law, received one or zero photon.

Therefore, a more accurate model of the noise to modify the way the spatial priors are involved does not help the algorithm FrIM-IMC to improve its performance at very low flux. This is because the control first needs to be improved. This is what we investigate in the next section thanks to a pseudo open-loop algorithm.

\section{Improving temporal behavior with a pseudo open-loop control}

In Sect. 2, FrIM-I provides a good Strehl, although the turbulent priors used in FrIM are not consistent with the statistics of the residual signal sensed in closed-loop. In Sect. 3, FrIM-IMC algorithm allows thanks to the internal model control to apply the turbulent priors on pseudo open-loop data, which make consistent the use of the turbulent statistics. However, the performance is strongly degraded. The pseudo open-loop control (POLC) of an AO system [6] provides a combination of these two approaches. Here, we have tested two different filters in the control :

$$
g(z)=\frac{\delta}{z^{2}-\alpha z-\beta} \quad \text { and } \quad g(z)=\frac{\delta}{z^{2}-z}
$$

following notations in [6]. The first filter is the one used by Gilles [6], where $\delta$ represents the integrator gain, and $\alpha=\beta=0.495$. The second one can be interpreted as a combination between a closed-loop integrator control and the IMC control studied above. The convergence curves of the long-exposure Strehl are compared in Fig. 5, with the filter of [6] on the left graph and the integrator POLC on the right.

Again, as the FrIM algorithm is used for the reconstruction step, the 4 different models of the noise introduced in Sect. 3 can be used with the POLC. They are all represented on the graphs of Fig. 5. 
Clémentine Béchet et al.: Very low flux adaptive optics using spatial and temporal priors
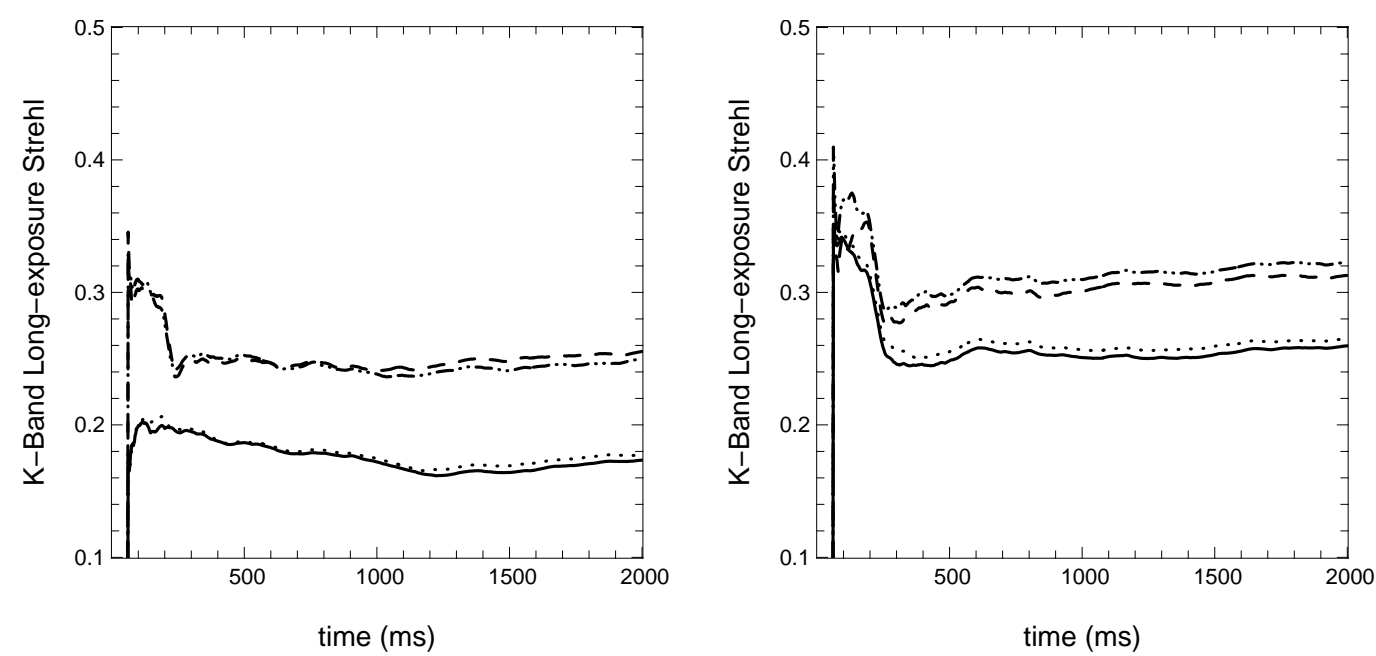

Fig. 5. Same axes as Fig. 3. Two different controls are compared. On the left : filter introduced in [6] for POLC. On the right : integrator POLC. As in Fig. 4, 4 noise models for FrIM are tested. Solid: uniform noise. Dotteddashed $: \gamma=0.1$. Dashed $: \gamma=0$. Dotted $: \gamma=1$.

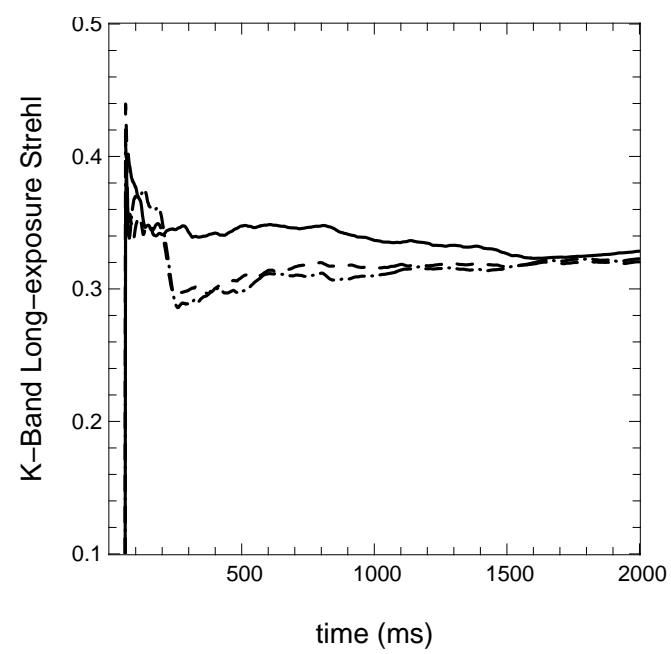

Fig. 6. Same axes as Fig. 3. Solid : MVM-I. Dashed : FrIM-I. Dotted-dashed : FrIM-POLC with integrator and $\gamma=0.1$.

First, analyzing the performance of the filter introduced in [6] (here on the left graph of Fig. 5), the uniform noise model (solid) and the case of $\gamma=1$ provide slightly lower Strehl than they do with FrIM-IMC control (18\% instead of $20 \%$, see Fig. 4). However, using a more accurate model of the noise, with $\gamma=0$ (dashed) or $\gamma=0.1$ (dotted-dashed), the Strehl converges now toward a higher value, $25 \%$. Even better in the simulated conditions here, the use of POLC with an integrator pushes the curves towards higher Strehl values (right graph of Fig. 5). Like with the other POLC, the use of a uniform noise or $\gamma=1$ is not the optimal way to use the spatial priors. A Strehl of $32 \%$ is reached when a factor $\gamma=0.1$ is used to weight the artificial measurements, compared to the data coming from sub-apertures with one photon.

Finally, Figure 6 summarizes the convergence of the best algorithms studied in low flux conditions. The dotted-dashed line still represents the FrIM-POLC algorithm with integrator and $\gamma=0.1$. Applying this control and this noise model, the performance is very similar to the one of a classical integrator 
control with FrIM reconstructor (dashed). It produces the same Strehl as the MVM-I algorithm (solid), although the convergence does not follow the same behavior. The two methods involving FrIM reconstructor here lead to a severe drop of $\sim 5 \%$ around $200 \mathrm{~ms}$, before slowly going up again. The MVM-I algorithm does not show such behavior. This difference may then come from the reconstructor, but a formal analysis of this is required to confirm it.

Running several other end-to-end simulations at very low flux, it has been possible to obtain the same long-exposure performance with MVM-I and FrIM-POLC algorithms as in the case presented in Fig. 6. However, the behavior of these two controls need to be formalized in order to be fully compared. Particularly, it is still not clear why the simple classical MVM-I algorithm is as efficient as a sophisticated reconstructor and controller (like FrIM-POLC) which uses an accurate statistics description for priors. Such study should be addressed in the future.

\section{Conclusion}

This paper shows several ways to involve the Fractal Iterative Method (FrIM) in a reconstruction and control algorithm for very low flux AO conditions. Simulations results are presented for an average of 1 photon per frame per sub-aperture. In such conditions, performance of FrIM with an internal model control at very low flux was found to be limited in Béchet et al. [3]. However, we have shown that choosing a pseudo open-loop control (POLC) with an integrator and optimizing the noise weighting according to the instantaneous flux is a solution to improve the performance. The reached Strehl is then the same as the one obtained by a classical command matrix with an integrator (MVM-I), but at a much lower computational cost thanks to the use of FrIM reconstructor. The new algorithm is obtained modifying the use of the spatial priors and the temporal aspects of the control, in order to adapt it to the very low flux conditions.

Beyond these first results and these comparisons, many questions remain open and need to be addressed in the future of this study. Although MVM-I and the POLC with the integrator can produce similar Strehl at low flux, they still contains two different controls and their stability and robustness properties should also be compared.

\section{References}

1. M. Tallon, R. Foy, Astron. Astrophys. 235, (1990) 549

2. J.D.H. Pilkington, Nature 330, (1987) 116

3. C. Béchet, M. Le Louarn, M. Tallon, É. Thiébaut, Adaptive Optics Systems: Proceedings of SPIE 7015, (2008) 70151H-70151H-9

4. M. Tallon, É. Thiébaut, C. Béchet, Adaptive Optics: Analysis and Methods/Computational Optical Sensing and Imaging/Information Photonics/Signal Recovery and Synthesis Topical Meetings on CDROM, (Optical Society of America, 2007) PMA2

5. C. Béchet, M. Tallon, É. Thiébaut, JOSA A. 26, (2009) 497

6. L. Gilles, Applied Optics 44, (2005) 993

7. C. Béchet, M. Tallon, É. Thiébaut, Advances in Adaptive Optics II : Proceedings of SPIE 6272, (2006) 62722U 